\title{
An evaluation of the perinatal mortality surveillance system in Gwanda District, Matabeleland South Province, Zimbabwe
}

\author{
P T Mundagowa, ${ }^{1}$ RN, MPH; P T Chimberengwa, ${ }^{2} \mathrm{MD}, \mathrm{MPH} ; \mathrm{E}$ M Chadambuka, ${ }^{1} \mathrm{RN}, \mathrm{MPH}$ \\ ${ }^{1}$ College of Health Sciences, Department of Clinical Research Centre, Africa University, Mutare, Zimbabwe \\ ${ }^{2}$ Ministry of Health and Child Care, Gwanda Provincial Hospital, Gwanda, Zimbabwe
}

Corresponding author: P T Mundagowa (mundagowap@africau.edu)

\begin{abstract}
Background. A preliminary review of perinatal mortality surveillance data (January - August 2017) suggested an under-reporting of perinatal deaths in Gwanda District, Matabeleland South.

Objective. To evaluate the effectiveness of the perinatal mortality surveillance system in Gwanda District, Matabeleland South, Zimbabwe. Methods. This descriptive cross-sectional study interviewed 50 healthcare workers employed in the district using pretested intervieweradministered questionnaires. The sample was drawn from 16 randomly selected healthcare facilities. Records for perinatal mortality cases were reviewed and data were analysed.

Results. Only $32 \%$ of healthcare workers knew the case definition of perinatal death. Approximately two-thirds of participants (68\%) knew who should complete notification forms and $\sim$ half (52\%) of the respondents cited fear of blame as the reason for a low perinatal death report rate in the district. Although $50 \%$ of participants reporting having participated in perinatal death reviews and $78 \%$ reporting using the data for planning community health programmes, there was no recorded evidence in support. Perinatal mortality case notification forms were not in stock at 13 of the 16 sampled healthcare facilities.

Conclusion. Poor knowledge of the perinatal mortality surveillance system was found among participants. The low reporting rate suggests that the system does not function effectively in the district. Healthcare workers feared blame, which suggests management intervention is required to create a trusted reporting environment.
\end{abstract}

S Afr J Child Health 2020;14(2)82-86. https://doi.org/10.7196/SAJCH.2020.v14i2.1659

Perinatal mortality refers to fetal deaths that occur after 28 weeks of gestation, death of a neonate with a birth weight $>1000 \mathrm{~g}$ and neonatal deaths that occur within the first 7 days post delivery ${ }^{[1]}$ The perinatal mortality rate (PMR) reflects the quality and use of antenatal, labour and postdelivery care available to women and neonates, ${ }^{[2]}$ and so is an indicator of the quality of healthcare available to mothers and infants. Accurate records of perinatal deaths can portray trends in causes and reveal opportunities for the prevention of the deaths. Such information can be useful because fetal deaths create considerable emotional and psychological distress for patients' families and clinicians. ${ }^{[3]}$ Perinatal mortality surveillance systems (PMSS) aim to gather data that can be used to identify areas in need of public health action and can assist in planning and monitoring programmes for the evaluation of infant health.

Globally, seven million perinatal deaths occur annually, which translates to a $7 \%$ global burden of disease. ${ }^{[4]}$ In global health monitoring mechanisms, perinatal deaths are largely neglected and poorly recorded, as concerns tend to focus on infant mortality. ${ }^{[5,6]}$ Millions of stillbirths are missed every year, most of which are unaccounted for by death certification and classification..$^{[7]}$ In high-income countries, the average stillbirth rate is low, at around 3.5 per 1000 live births. ${ }^{[8]}$ However, Zimbabwe recorded a rate of 29 deaths per 1000 live births in 2014. ${ }^{[9]}$ The report on the National Assessment of Maternal and Perinatal Death Surveillance and Response Implementation in Zimbabwe (2017) revealed that of 16 facilities, only six consolidated data on neonatal mortality and five facilities acknowledged that they did not conduct regular perinatal death audits. ${ }^{[1]}$ The report also found that audits tend to focus on maternal mortality rather than perinatal mortality. For Zimbabwe to reduce the neonatal mortality rate from the 2014 baseline of 29 deaths per 1000 live births to 20 by 2020, a more active and sensitive PMSS is needed. Fig. 1 shows the current reporting structure and timelines for PMSS in Zimbabwe.

A preliminary review of perinatal mortality surveillance data from Gwanda Provincial Hospital (the biggest referral hospital in Matabeleland South province) showed that 33 perinatal deaths were recorded between 1 January and 31 August 2017. However, only

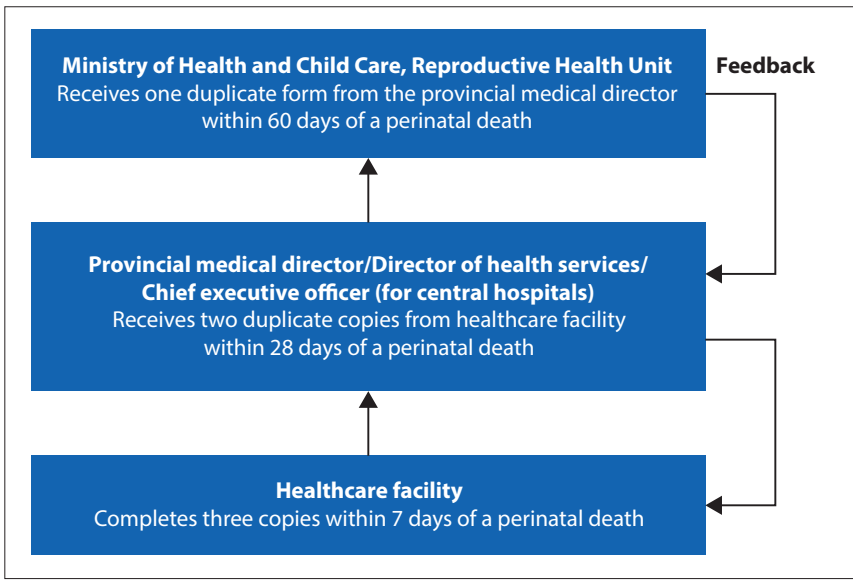

Fig. 1. Flow diagram showing the reporting structure and timelines for the perinatal mortality surveillance system in Zimbabwe. Adapted from Guidelines for Maternal and Perinatal Death Audits in Zimbabwe, Ministry of Health and Child Welfare. ${ }^{[10]}$ 
$10(30 \%)$ of these deaths were reported. Eight out of these 10 deaths were notified after more than 60 days and all the submitted forms had some missing information. However, the completeness of these 10 perinatal death reporting forms was fairly good, on average, at $89.4 \%$. According to the Multiple Indicator Cluster survey of 2014, the neonatal mortality rate of the Matabeleland South province was 35 deaths per 1000 live births, higher than the national rate of 29 deaths per 1000 live births. ${ }^{[9]}$ It was against this background that we sought to evaluate the perinatal mortality surveillance in Gwanda District.

\section{Methods \\ Research design}

A descriptive cross-sectional study was used to evaluate the PMSS. The study population consisted of healthcare workers (nurses and doctors). Perinatal mortality registers, delivery registers and perinatal death reports were also reviewed. The study was conducted in Gwanda District in the Matabeleland South province, Zimbabwe.

The study used the surveillance system described in the Guidelines for Maternal and Perinatal Death Audits in Zimbabwe issued by the Ministry of Health and Child Welfare (2013). ${ }^{[10]}$ The effectiveness of a surveillance system is guided by its attributes, ${ }^{[1]]}$ identified as follows in this study:

- Usefulness: Contribution to preventing and controlling adverse health-related events, including an improved understanding of the public health implications of such events

- Simplicity: The system's structure and ease of operation

- Acceptability: The willingness of persons or organisations to participate in the system, including those who operate the system, report cases and use the data obtained

- Stability: The ability of the system to collect, manage and provide data without failure

- Sensitivity: The proportion of cases of a health-related event detected by the system and the ability to monitor changes over time

- Timeliness: Reflects the delay between steps in a surveillance system and the availability of information of the health-related event under surveillance when needed.

\section{Sampling}

The sample size was calculated according to Dobson's formula: $n=Z^{2} p q / e^{2}$, where $Z$ is the statistic used for the $95 \%$ confidence interval. According to the attendance register of the district's perinatal meetings, about $97 \%$ of healthcare workers had attended at least one perinatal meeting, and $p$ (the estimated proportion of participants who had attended at least one such meeting) was therefore taken as 0.97 . The variable $e$ represents the desired precision. The minimum required sample size was calculated as 45 .

Gwanda District has 30 health facilities, which include one provincial hospital, two mission hospitals, nine government rural health centres, 13 rural district council clinics and five private clinics. Multistage sampling was used to select health facilities to be sampled. The provincial hospital and the two mission hospitals were purposively selected and 13 clinics were randomly selected using the lottery method. Five randomly selected healthcare workers from the maternity wards of each hospital were selected for the study. At the clinics, all nurses on duty on the day of data collection were selected for the study. The nurses in charge, district nursing officer and the district medical officer were also purposively recruited into the study as key informants.

\section{Data collection and analysis}

Data were collected using questionnaires and checklists, which were pretested for validity and reliability at a non-participating clinic. Checklists and interviewer-administered questionnaires were also used to collect data during record reviews and interviews. Healthcare workers were interviewed to determine their knowledge of the operation of the PMSS and to assess some of the system attributes. Completed perinatal death reports and registers for the period 1 January 2016 - 30 June 2017 were reviewed to assess data quality, simplicity, completeness and timeliness of the reporting system.

The Epi Info software package (Centers for Disease Control and Prevention, USA) and Excel (Microsoft Corp., USA)were used to analyse the data. Quantitative data were expressed as frequencies and means, whereas qualitative data were analysed manually. Observed data were categorised or coded to facilitate statistical analysis.

\section{Ethical considerations}

Permission for the study was granted by the Research Ethics Committee at the Africa University (ref. no. 304/17) and the provincial medical director (Matabeleland South).

\section{Results}

A total of 50 participants were recruited for the study (Table 1). Almost three-quarters of the sample were female $(n=36 ; 72 \%)$. The median duration of in-practice experience was 8.5 years. Primary care nurses represented approximately half of the sample $(n=26 ; 52 \%)$ and midwives made up approximately a third of the sample ( $n=17 ; 34 \%)$.

Table 2 shows participants' knowledge of the features of PMSS. Approximately a third $(n=16 ; 32 \%)$ of participants were able to define a perinatal death and the majority $(n=35 ; 70 \%)$ could identify the targeted age group. Most participants $(n=41 ; 82 \%)$ correctly identified the number of case forms to be completed and all but one ( $n=49 ; 98 \%)$ correctly noted the destination of the completed forms. Healthcare workers cited a lack of knowledge with regard to the reporting procedure and suggested regular attendance of perinatal meetings and audits held at hospitals as a means to build capacity among district healthcare workers.

The major reasons for not reporting perinatal deaths in Gwanda District appeared to be fear of blame and reprimand upon signing personal identification details on submitted case forms $(n=26$; $52 \%)$ and the necessary perinatal death forms not being available at healthcare centres $(n=19 ; 38 \%)$ (Fig. 2$)$.

\section{Attributes of the perinatal mortality surveillance system Usefulness}

A large proportion of participants $(n=46 ; 92 \%)$ reported that PMSS data are analysed at district level, whereas $78 \%(n=39)$ reported use of the information at a local level. However, there was no evidence of case analysis for any centres other than Gwanda Provincial Hospital and Mtshabezi Mission Hospital, which had minutes of perinatal death audits on record. Half of the sample $(n=25)$ reported having had a discussion or audit related to PMSS, although recorded minutes were available only at Gwanda Provincial Hospital and the Mtshabezi Mission Hospital.

Most participants $(n=44 ; 88 \%)$ perceived PMSS to be useful at district level. Some participants $(n=20)$ reported having used PMSS for public health initiatives in the district, with six and three reporting using the information in awareness campaigns about perinatal deaths and implementing active case searches, respectively. However, no records of such campaigns or active searches were found.

\section{Simplicity}

The two-page form used for reporting a perinatal death consists of six fields (Table 3). Nineteen participants reported to have filled out the notification form before, with responses from 11 of these noting 


\begin{tabular}{ll}
\multicolumn{2}{l}{ Table 1. Baseline characteristics of study participants $(\mathbf{N = 5 0})$} \\
\hline Characteristic & $n(\%)^{*}$ \\
\hline Sex & $36(72)$ \\
Female & $14(28)$ \\
Male & $8.5(4-10)$ \\
In-practice experience (years), median (IQR) & \\
Designation & $6(12)$ \\
Registered general nurse & $17(34)$ \\
Midwife & 0 \\
State-certified nurse & $26(52)$ \\
Primary care nurse & $1(2)$ \\
Doctor & \\
Health institution (N=16) & $5(31)$ \\
Government & $9(56)$ \\
Councils (rural and municipal) & $2(13)$ \\
Mission & \\
${ }^{*}$ Unless otherwise specified. &
\end{tabular}

Table 2. Study participants' knowledge of operational aspects of the perinatal mortality surveillance system $(N=50)$

\begin{tabular}{ll}
\hline Aspect correctly identified & $n(\%)$ \\
\hline Definition of perinatal death & $16(32)$ \\
Targeted age group & $35(70)$ \\
Person responsible for completing forms & $34(68)$ \\
Register for entering deaths & $17(34)$ \\
Number of forms to be completed & $41(82)$ \\
Destination of completed forms & $49(98)$ \\
Timeline for the notification & $17(34)$
\end{tabular}

Table 3. Completeness of data on perinatal death notification forms $(N=40)$

\begin{tabular}{|c|c|c|}
\hline Field & Description & $\begin{array}{l}\text { Number of forms with fully } \\
\text { completed sections, } n(\%)\end{array}$ \\
\hline $\mathrm{A} 1$ & Details of institution & $35(88)$ \\
\hline A & Demographics & $39(98)$ \\
\hline B & $\begin{array}{l}\text { Maternal medical } \\
\text { conditions }\end{array}$ & $29(73)$ \\
\hline $\mathrm{C}$ & $\begin{array}{l}\text { Antenatal care, labour } \\
\text { and delivery }\end{array}$ & $30(75)$ \\
\hline $\mathrm{D}$ & Stillbirths only & $35(88)$ \\
\hline $\mathrm{E}$ & Neonatal deaths only & $31(78)$ \\
\hline $\mathrm{F}$ & Delays & $35(88)$ \\
\hline $\mathrm{F} 1$ & $\begin{array}{l}\text { Health worker } \\
\text { identification }\end{array}$ & $37(93)$ \\
\hline $\mathrm{F} 2$ & $\begin{array}{l}\text { Head of institution } \\
\text { details }\end{array}$ & $26(67)$ \\
\hline
\end{tabular}

that it took 10 - 20 minutes to complete the form. A need for training on PMSS and the process for completing the notification form was reported by 29 participants as a step towards improved service.

All reported case forms had some missing data; details of the head of the institution (section F2) were fully completed least often (67\%). On average, notification forms showed an $83 \%$ completion rate. The completeness of data in the various fields is summarised in Table 3.

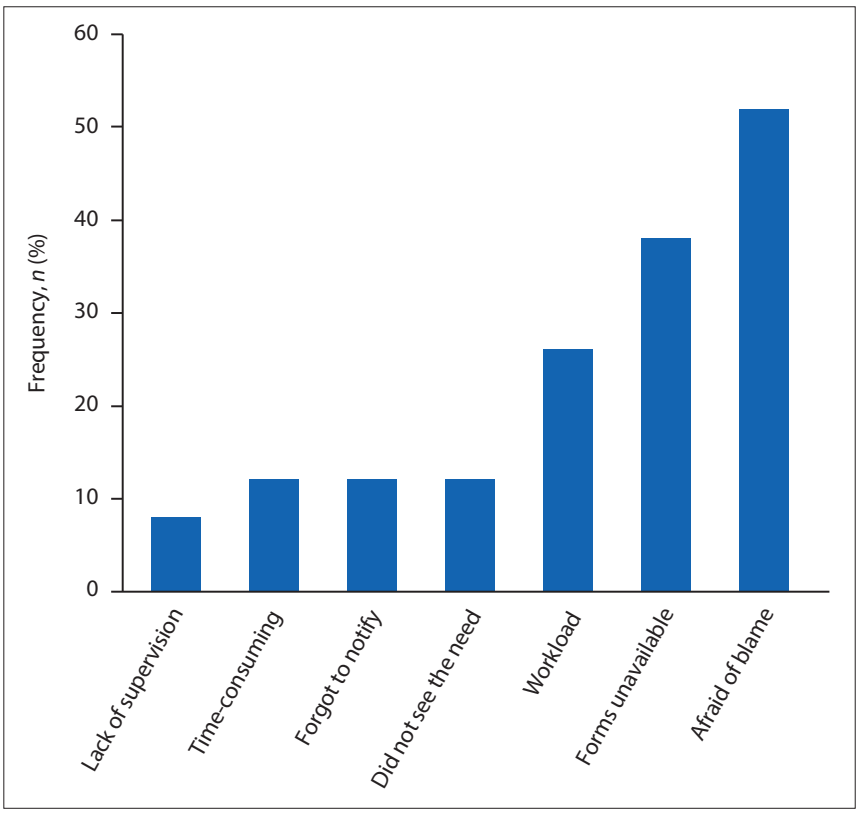

Fig. 2. Frequencies of reasons for low notification of perinatal deaths, Gwanda District, January 2016 - June 2017.

\section{Acceptability}

Almost all participants (96\%) reported that they considered it their duty to report perinatal deaths and were willing to continue to participate in the PMSS. However, evidence of reporting a perinatal death was found only at three of the 16 healthcare centres surveyed. Nine of the clinics visited did not record a perinatal death during the period under study. Fig. 2 shows the results of reasons for low notification of perinatal deaths in the district.

\section{Stability}

Forms for reporting a perinatal death were available at only three of the surveyed facilities. None of the surveyed institutions had a mechanism in place for checking the timeliness of reporting. Six rural health centres were without functional mobile phones and four of these reported mobile network challenges that affected communication. Institutions that did report perinatal deaths had received no feedback from the relevant health authorities. The surveillance system was found to be unstable. Some participants reported forms being lost along the notification chain.

\section{Sensitivity and timeliness}

According to the report on maternal and perinatal death surveillance in Zimbabwe, the PMSS aim to identify all stillbirths and early neonatal deaths at a health facility or in the community. ${ }^{[1]}$ The official guidelines state that a healthcare facility should submit three notification forms to the district office within 7 days of a perinatal death. The district office then has to submit two forms to the provincial office within 28 days of the perinatal death and the national office has to receive one copy within 60 days of the perinatal death (Fig. 1).

Participants reported that the surveillance system fails to capture stillbirths and neonatal deaths that occur in communities, leading to an under-reporting of cases. The failure stems mainly from healthcare workers' limited understanding of the notification system and a lack of logistical resources to follow up cases in a community. None of the notification forms was submitted within the stipulated 
timeframes, with 25 (63\%) of the forms being received by district offices more than 4 months after the perinatal death had occurred. None of the reporting centres received feedback from the district or provincial offices. Of the 117 cases that occurred during the period of review, only 40 were reported.

\section{Discussion}

Our findings show that healthcare workers in Gwanda District generally had little knowledge about the purpose and functioning of PMSS. Adequate knowledge of the surveillance system and its functioning among healthcare workers is crucial for accurate detection of perinatal deaths and prompt response at the primary level. A lack of knowledge by healthcare workers may contribute to their failing to report perinatal deaths, as found in a Tanzanian study. ${ }^{[12]}$

Our study showed that only $32 \%$ of healthcare workers were able to clearly define a perinatal death. This can negatively affect the effectiveness of the surveillance system, as the number of cases will not be accurately reported. A study among healthcare workers in Atlanta, USA, showed that insufficient knowledge regarding the surveillance system led to incomplete and vague data being reported. ${ }^{[13]}$ Our findings suggest a similar outcome: healthcare workers' poor knowledge of the surveillance system culminated in forms not being adequately completed and a low report rate, which impede the effectiveness of surveillance activities. Some participants confused perinatal death and maternal death, and only $32 \%$ of the participants could clearly define a perinatal death. Such variation in interpretation may lead to inconsistencies in reporting. ${ }^{[14]}$

Healthcare workers in Gwanda District were willing to participate in the surveillance programme as acceptability was $96 \%$. The observation suggests that understanding how data gathered by a surveillance system are used can motivate healthcare workers to participate in the surveillance system. Lenaway and Ambler ${ }^{[15]}$ reached a similar conclusion in a study that evaluated a school-based influenza surveillance system: both school authorities and nursing staff were willing to participate in surveillance activities because the programme was perceived as beneficial by participants.

The loss of notification forms along the reporting chain contributes to inaccurate statistics, and lack of feedback from the relevant health authorities can potentially reduce healthcare workers' enthusiasm to participate in the surveillance system. Lack of interest by healthcare workers can eventually result in under-reporting, which is a barrier to the quality of data collected, as concluded in a similar study in the USA. ${ }^{[16]}$ The lack of feedback identified in the Gwanda District was consistent with poor feedback mechanisms noted at national level in the Guidelines for Maternal and Perinatal Death Audits in Zimbabwe. ${ }^{[10]}$

Participants who had experience in dealing with perinatal deaths, such as hospital nurses, found the PMSS simple to use. However, participants who had little experience of such cases viewed the system as difficult to understand. This suggests that allocating resources at district level to familiarise healthcare workers with the legislative guidelines related to reporting maternal and perinatal mortality will be beneficial. The high number of healthcare workers expressing fear of blame upon completing the notification forms may be attributed to the fear of backlash associated with maternal or perinatal death reviews, despite the notification form highlighting that the information will not be used for medicolegal investigation. It is therefore important that supervisors ensure confidentiality and anonymity during an inquiry of perinatal deaths, as cited in the national guidelines. ${ }^{[10]}$

Almost all (98\%) participants accepted the PMSS as a source of useful health indicators for the district. This is similar to findings from a study in Atlanta, USA, where a high proportion of respondents accepted the surveyed surveillance system because of its perceived outputs. ${ }^{[13]}$

All participants who had completed the notification form before found the two-page tool easy to complete. In contrast, Tachiwenyika et al. ${ }^{[17]}$ reported that an earlier version of the form, which focused on the determinants of perinatal mortality, was found difficult to complete. The notification form was subsequently reviewed and simplified to the format currently in use. Simplicity in completing the form helps to improve functionality of the system, considering the heavy workload and multiple administrative registers already faced by healthcare workers at rural healthcare centres and maternity wards.

Stability is an important attribute for the functionality of a surveillance system. The functioning of the PMSS was not stable in Gwanda District, as stationery was not readily available at most $(88 \%)$ of the surveyed healthcare centres. Similarly, a Tanzanian study reported that $67 \%$ of the necessary tools for data collection were unavailable at rural healthcare centres. ${ }^{[18]}$ A lack of essential resources, such as notification forms, affects the stability of a public health surveillance system. Studies from Tanzania ${ }^{[12,19]}$ have also noted that challenges related to infrastructure systems and workload (e.g. poor communication systems, inadequate laboratory facilities, difficult access to healthcare facilities and overburdened staff) can negatively affect the stability of a surveillance system such as for perinatal data.

A low level of consistency and monitoring of PMSS was found in Gwanda District, as seen in the low report rate for perinatal deaths. This can stifle efforts to prevent miscarriages and neonatal deaths. For PMSS to be effective, the system has to allow for consistent, prompt and effective reporting, which can trigger action to prevent future infant fatalities. ${ }^{[12]}$ Owing to under-reporting and lack of representativeness of PMSS in Gwanda District, the available data were of poor quality and could not reliably contribute to an estimation of the burden of perinatal mortality in the district. Continued efforts to educate healthcare workers in detecting and reporting perinatal deaths within the system is recommended.

\section{Study limitations}

Self-reporting as used in this study may introduce social desirability bias. Using convenience sampling in data collection could be a source of bias in this study.

\section{Conclusion}

Our findings showed that although healthcare workers in the Gwanda District had some knowledge of PMSS, challenges associated with the notification process compromised the effectiveness of the system. The system was acceptable to the majority of healthcare workers in the district, but its functioning was found to be unstable. Limited data were available for estimating the burden of perinatal deaths or determining the trend of perinatal deaths in the district. Fear of blame was cited as a barrier to reporting and managers are urged to foster a trusted reporting environment. Records of activities such as awareness campaigns that flowed from recorded data can help to encourage others to contribute to accurate reporting, thereby improving the usefulness of the surveillance system. Further studies on the functioning of PMSS at the provincial level are recommended.

Declaration. This manuscript was submitted in partial fulfilment of the requirements for a Master of Public Health degree. 
Acknowledgements. We thank the provincial medical director (Matabeleland South) for permission to conduct this study. We also acknowledge the participating healthcare workers of Gwanda District. Author contributions. PTM conceptualised the study, compiled the study protocol, collected data and wrote the manuscript. PTC and EMC reviewed the protocol and the manuscript.

\section{Funding. None.}

\section{Conflicts of interest. None.}

1. Maternal and Child Survival Program. Assessment of maternal and perinatal death surveillance and response implementation in Zimbabwe. November 2017. https://www.mcsprogram.org/resource/assesment-maternal-perinatal-deathsurveillance-response-implementation-zimbabwe/

2. Abdel AA, Elgessim ME, Tahah E, Adam GK. Factors associated with perinatal mortality in Kassala, Eastern Sudan: A community-based study 2010-2011. J Trop Pediatr 2014;60(1):79-82. https://doi.org/10.1093/tropej/fmt075

3. Silver RM. Fetal death. Obstet Gynecol 2007;109(1):153-167. https://doi. org/10.1097/01.aog.0000248537.89739.96

4. Partnership for Maternal, Newborn and Child Health. Knowledge Summary \#27 - Death reviews: Maternal, perinatal and child. Geneva: PMNCH/WHO, 2013. https:/www.who.int/pmnch/knowledge/publications/summaries/ks27/en/

5. Allanson ER, Tuncalp O, Gardosi J, et al. Giving a voice to millions: Developing the WHO application of ICD-10 to deaths during the perinatal period: ICD-PM. BJOG 2016;123(12):1896-1899. https://doi.org/10.1111/1471-0528.14243

6. Duke W, Williams L, Correa A. Using active birth defects surveillance programs to supplement data on fetal death reports: Improving surveillance data on stillbirths. Birth Defects Res A Clin Mol Teratol 2008;82(11):799-804. https://doi. org/10.1002/bdra.20526

7. Lawn JE, Blencowe H, Oza S, et al. Every newborn: Progress, priorities, and potential beyond survival. Lancet 2014;12(384):189-205. https://doi.org/10.1016/ s0140-6736(14)60496-7

8. Flenady V, Wojcieszek AM, Middleton P, et al. Stillbirths: Recall to action in highincome countries. Lancet 2016;387(10019):691-702. https://doi.org/10.1016/ s0140-6736(15)01020-x
9. UNICEF. Extended Analysis of Multiple Indicator Cluster Survey (MICS) 2014 Nutrition, Health and Water and Sanitation. Harare: UNICEF, 2016.

10. Ministry of Health and Child Welfare [Zimbabwe]. Guidelines for Maternal and Perinatal Death Audits in Zimbabwe. Harare: MoHCW, 2013.

11. Centers for Disease Control and Prevention. MMWR Recommendations and Reports. Updated Guidelines for Evaluating Public Health Surveillance Systems. MMWR 2001;50(RR13):1-35. https://www.cdc.gov/mmwr/preview/mmwrhtml/ rr5013a1.htm

12. Kilonzo A, Kouletio M, Whitehead SJ, Curtis KM, McCarthy BJ. Improving surveillance for maternal and perinatal health in 2 districts of rural Tanzania. Am J Public Health 2001;91(10):1636-1640. https://doi.org/10.2105/ajph.91.10.1636

13. Azofeifa A, Yeung LF, Duke CW, Gilboa SM, Correa A. Evaluation of an active surveillance system for stillbirths in metropolitan Atlanta. J Registry Manag 2012;39(1):13-18

14. Public Health Agency of Canada. Canadian Perinatal Health Report, 2008 Edition. Ottawa: Public Health Agency of Canada, 2008. http://www.publichealth. gc.ca/cphr/ (accessed 31 July 2019).

15. Lenaway DD, Ambler A. Evaluation of a school-based influenza surveillance system. Public Health Rep 1995;110(3):333-337.

16. Duke W, Gilboa SM. The utility of using an existing birth defects surveillance program to enhance surveillance data on stillbirths. J Registry Manag 2014;41(1):13-18.

17. Tachiweyika E, Gombe N, Shambira G, Chadambuka A, Mufuta T, Zizhou S Determinants of perinatal mortality in Marondera district, Mashonaland East Province, Zimbabwe, 2009: A case control study. Pan Afr Med J 2011;8:7. https:// doi.org/10.4314\%2Fpamj.v8i1.71054

18. Rumisha SF, Mboera LE, Senkoro KP, Gueye D, Mmbuji PK. Monitoring and evaluation of integrated surveillance and response in selected districts in Tanzania. Tanzan Health Res Bull 2007;9(1):1-11. https://doi.org/10.4314/thrb. v9i1.14285

19. Mboera LEG, Rumisha SF, Magesa SM, Kitua AY. Utilisation of health management information system in disease surveillance in Tanzania. Tanzan Health Res Bull 2001;3(2):15-18. https://doi.org/10.4314/thrb.v3i2.14213 